\title{
A New Educational Mobile Devices Platform for Social Inclusion in Tanzania
}

Fredrick Mtenzi

Technological University Dublin, Fredrick.Mtenzi@tudublin.ie

Follow this and additional works at: https://arrow.tudublin.ie/scschcomart

Part of the Computer Engineering Commons

\section{Recommended Citation}

Mtenzi, F.J. (2016) A New Educational Mobile Devices Platform for Social Inclusion in Tanzania, Journal of ICT Research in Africa and the Middle East, doi: 10.4018/JICTRAME.2016070105

This Article is brought to you for free and open access by the School of Computer Sciences at ARROW@TU Dublin. It has been accepted for inclusion in Articles by an authorized administrator of ARROW@TU Dublin. For more information, please contact arrow.admin@tudublin.ie, aisling.coyne@tudublin.ie,gerard.connolly@tudublin.ie.

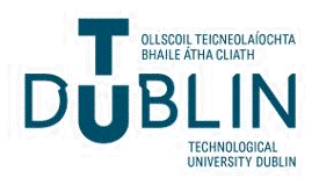




\title{
A New Educational Mobile Devices Platform for Social Inclusion in Tanzania
}

\author{
Fredrick Japhet Mtenzi \\ School of Computing, College of Sciences and Health, \\ Dublin Institute of Technology, \\ Dublin, Ireland. \\ Fredrick.Mtenzi@dit.ie
}

\begin{abstract}
It is evident that advances in technology has led to improvement in societal wellbeing. In this paper we demonstrate how mobile phones are used in providing reliable and quality education to students in disadvantaged areas of Tanzania. The main contribution is on leveraging on the success that Tanzania has had on using mobile banking to the un-banked population. These lessons are adapted to the education sector, where clever/smart integration of existing disruptive technologies such as mobile phones and social networks are be used to provide access to high quality educational contents. Further, the paper shows how educational content can be localized/adopted with a view of empowering teachers and the educational needs of students. The central piece of the proposed system is the ability to add, share and reduce the amount of redundancy. Finally, we report on the implementation of the system in a community project in rural Tanzania
\end{abstract}

Keywords-social inclusion; educational platform, disruptive technologies, social networks, national research networks, mobile network operator;

\section{INTRODUCTION}

In the last two decades, the proliferation of mobile phone networks has transformed communications in the world and the sub-Saharan Africa is no exception. It has also allowed subSaharan Africa to skip a few generations of technology development and jump right to the digital age. According to the research done by Pew Research the ownership of mobile phones in sub-Saharan Africa is comparable to developed countries [1]. However, in another research Pew Research show significant gender and educational differences in mobile phone ownership and usage [2]. While it is argued that most people from these countries afford owning mobile phone, the cost of running a mobile phone is still significantly higher compared to their annual earnings. These tariffs will continue to be high until governments enact legislations that will allow them to curb/control the profit motives of the mobile network operators.

The use of mobile phones has gone beyond the ability to make phone calls. Nowadays a myriad of value added services are available even in low end mobile phones. These value added services have stimulated innovations in all walks of life from healthcare to agriculture and education. For example, mobile phones have provided one of the most disruptive forces that the payments industry has seen in Tanzania. Mobile phones are unleashing profound changes and innovation in the development of simple, personal and always available mobile payment schemes. The advent of mobile payment schemes has led to the emergence of new business models and players, such as mobile payment providers, aggregators and agents. It is no wonder that consumers throughout Tanzania want to use mobile payment schemes because they are convenient, personal, on demand and are seamlessly integrated into everyday life. Further, these mobile payment schemes are challenging the very existence of traditional financial services such banks.

The majority of Tanzanians are and will continue using cash as a way of paying for services for a foreseeable future. However, mobile payment schemes are now a feasible way of reducing the amount of cash which people and the society as a whole carry for paying for services. This is clearly demonstrated by the success of first generation mobile payment schemes such as M-Pesa, TigoPesa, Airtel Money etc. The second generation is a group of mobile payment aggregators consisting of the likes of DataVision International Limited [3], Selcom Wireless [4], Maxcom Africa [5]. For example, DataVision International Limited is now offering customized services such as Ada-Lipa. The availability of all these services is providing a fertile ground for further innovation in mobile payment schemes which can be extended using the same aggregation platform; the possibilities are surely endless.

There are a number of communication technologies employed in mobile payment schemes. These technologies include SMS/Unstructured Supplementary Service Data (USSD) due to its compatibility with any mobile phone and ease of implementation, used mainly in developing countries. Other communication technologies are near field communication (NFC), Quick Response (QR) codes, Bluetooth Low Energy (BLE), sound wave and Magnetic Secure Transmission (MST). In applications areas such as education or healthcare uses other technologies such as instant messaging, web email and podcasts/vodcasts [6].

Mobile payment schemes have and are going to form one pillar of financial transactions in Tanzania. The mobile payment if used innovatively will guarantee the success of eGovernment, m-Government, healthcare and education in Tanzania. The future of mobile payment schemes will be 
littered with new technologies. Some of these hold great promise for example, wearable technologies allow to make payments via an electronic device worn on your body such as a watch. Peer-to-Peer Payments (P2P) where consumers can pay people directly via electronic channels, including social media. Virtual currencies allow users to transact with currency based on cryptographic algorithms. Cloud based mobile payment might lead to the development of mobile payment systems using a cloud based architecture.

Mobile phones have not only revolutionized the way we communicate but also, in many classrooms around the globe, mobile technologies are increasing the power of learning. When mobile phone are an integral part of teaching they can reduce stationary usage which is one of the major costly items for students. Further, the use of mobile phone in education will encourage and accelerate the use of the cloud. Mobile phones are affordable for most people and provide greater opportunities to foster collaborative learning.

Mobile phones have the potential of creating greater access to education around the world. It is expected to break even the gender barriers and social stigma. The project reported in this paper was done to empower young girls in Tanzania by improving their technical literacy in programming and the can do attitude. Further, to increase their confidence and selfesteem and gain marketable skills. This project will serve as one of the launch pad for Tanzanian girls to explore and excel in both entrepreneurship and technological innovations. All these initiatives clearly show that Technology is a tool for transforming the way we learn.

It is interesting and encouraging to witness the emergence of home grown talent leading the innovation in developing mobile payment and educational solutions that address Tanzania needs. The trajectory taken in developing these solutions, provides a space to learn and adapt similar initiatives undertaken in other parts of the world. There is a new ecosystem of companies in Tanzania devoted to developing innovative mobile solutions. These solutions are now being adopted and used in public and private sector, some examples are reported in [7].

\section{BACKGROUND}

Many countries are currently planning, designing and investing heavily in projects that use mobile phones for education. These projects will continue to fail or not be sustainable unless the following three concepts are incorporated: teachers/instructors play the key role in determining the success and failure of such projects; change is a year's long process and not a one-time event; and teachers need on-going support to adopt the mobile phone technology and should be treated as stakeholders in the innovationadoption process [8]. It is amazing how projects from small to big do not plan or completely forgot to train and involve teachers as stakeholders.

The assumption that end users can just use mobile phones is not correct. The results from this project strongly suggest that for effective and efficient use of mobile phones, end-users must be trained. This reduces frustration and makes learning fun, which was one of the main aims of the project [8].
There are a lot of research on the benefits of using ICT in education and evidence shows that it is the case. However, it is also true that use of technology does not eliminate inequalities, in some cases it makes them worse [9]. There are two reasons why technology alone does not address the inequality problem. First, the rich can afford always afford more and better technology. Second, elimination of digital divide does not eliminate socio-economic divides.

Another criticism which has been leveled on the use and ownership of mobile devices is at what age children should be allowed to use mobile phones. Anecdotal evidence suggest that children as young as four years old are allowed to use mobile devices. The questions that we should be asking as a society are what age is too young to have a phone and how owning one helps or harms kids [10]. These and other similar ethical questions demand more research being done so as to advance our understanding on the issue.

Owning and using mobile devices among young children may lead to them being connected to applications such as social media which can exacerbate problems like bullying, harassment and FOMO (fear of missing out) [10]. It has been reported that there are schools and households that have banned the use of mobile devices. This seem to tally with evidence reported in [11] on how performance of students increased when they banned the use of mobile phones in school. Mobile phones can be a source of great disruption in workplaces and classrooms, as they provide individuals with access to texting, games, social media and the Internet. Given these features, mobile phones have the potential to reduce the attention students pay to classes and can therefore be detrimental to learning.

Some studies suggest excessive time spent using screens, including cellphones, can affect children and youth adversely, stunting emotional and cognitive growth, warping a young person's perspective on social norms and causing health problems such as poor eating and sleeping habits [12], [13] and [14].

Despite the common theme that seem to emerge from all the negative impact of mobile devices use in education, it is true as well that when incorporated into the curriculum and being put to a well-defined use, mobile devices have the potential to improve student outcomes. The literature is littered with examples of improved performance and increased participation among students from different background and income levels.

There are a few examples of paid mobile phone educational products in Tanzania and they have adopted some form of micropayment experience from mobile banking. While paying for educational services may sound normal, it is expensive for a normal Tanzanian who must pay for expensive connectivity costs as well. To make matters worse, some educational services must be paid using a credit card or PayPal account making their use beyond the reach of most Tanzanians. Thus digital divide can be perpetuated between those who have these credit cards [15].

In order to achieve universal social inclusion free access must be encouraged. The blockchain concepts from Bitcoin can 
be adopted to mobile phone in education to allow for peer to peer micro secure transactions that does not depend on central monitoring authority. Examples of free access mobile phone educational products include Nokia Mobile Mathematics which offers over 9000 exercises, mapped to high school level, and can be accessed by any person with a data and browser-enabled device [16] and Tanzania Online Virtual Library [17]. The Nurturing Minds [18] project in Morogoro, helps educate Tanzanian girls who are poor, marginalized and at-risk of becoming involved in exploitative forms of child labour. It supports the residential Secondary Education for Girls' Advancement (SEGA) School for motivated Tanzanian girls who otherwise are unable to attend school due to extreme poverty.

Use of mobile phones in education is also widespread in other countries in sub-Saharan Africa. For example, the Yoza Project [19] demonstrates the potential of mobile publishing for supporting teen reading and writing in South Africa and Kenya. SMS as a mobile phone application is currently being widely used in education. For example, the SMS learning application from Edimpact in India uses cloud assessment application at backend and the mobile SMS technology as frontend [20].

When mobile phones are used in education they encourage problem solving to end users. For example, if the user is playing an educational game in order to proceed from one level to the next must overcome a challenge or solve a problem. They also allow comprehensive feedback to be given to end users. This feedback can be stored for long time usage and analysis to improve the quality and delivery of the content. The most appealing feature of this feedback is its personalization and timely fashion.

The question is why we should design another educational networks when there are existing well established national research and education network (NRENs), Facebook and Google frameworks. The most important reasons is the cost and bandwidth availability. The ideas discussed in this paper were tested in the project that was implemented at Mtwango CoderDojo Project.

The mobile phone ability to multitask is one of the attractive features of the device. However, multitasking in most cases comes at the cost of distractibility when performing other tasks such as learning using a mobile phone. Distraction from mobile phone can viewed from three major aspects: distraction sources (ring of mobile phone, texting, and social applications), distraction targets (reading and attending), and distraction subjects (personality, gender, and culture) [21]. A lot of studies have concluded that multitasking in mobile phones does affect learning.

The value of games in education has been researched and documented in a lot of research outputs. Mobile phones lend themselves nicely to providing appropriate educational games. All the necessary trappings can be included in mobile phones games such as monitoring, controlling, feedback and evaluation. The concern of students spending most of their time in games can be easily controlled by using timeout and requiring additional knowledge to keep on playing or solving a problem to proceed to the next level. Further, clever connectivity protocols can lead to team based learning, where students work in groups to solve specific problems and in turn have fun and compete in a game. These students can be anywhere and can take part in the game when they have time.

It is important to acknowledge the fact that games are designed by people who understands technology and not pedagogy. Therefore educational game designers have paid close attention to the development of entertaining experiences for end users. Experts point out, an overwhelming number of projects do not have rigorous pedagogical foundations. Additionally, many existing educational game design frameworks have a one-size fits all approach [22]. Modern games have added the element of both auditory and kinesthetic stimulation, intended to help achieve a greater retention of the learning material [23]. It is expected that the next generation of educational mobile phone games will incorporate the entertainment factor and pedagogy.

It is easy to update online teaching materials and can provide filtering capabilities to ensure that illegal and harmful content is not accessible from any school designated mobile phone. The possibility of being able to monitor is important if schools need to review if phones are being used for their intended purpose and for accountability purpose.

There are three ways using mobile phones in education. The first way is to use mobile phone to only access information. The second way of using mobile phone is to allow the end-user to provide information. In the third way interaction occurs in various ways creating collaborative learning communities [24]. The work reported in this paper is based on the third type of usage which pose a new way to build collective knowledge and develop end-users collaboration skills.

Educational uses of mobile phones include [25]:

- Enables curricular based learning activities, for example, interactive quizzes, puzzles, mathematical problems etc., to be sent to mobiles

- $\quad$ Promotes the use of voice, data and video among peer-to- peer mentors and study groups

- Tailors learning for students to suit their learning styles. For example, students with special educational needs, by providing learning material with text or audio for students with hearing impairments

- Improves literacy and numeracy levels among students by providing personalized lessons

- Facilitates collaborative and project based learning

- Provides the capacity to access Internet resources, for example, revision notes and news updates

- Converges with other technologies, such as laptops and tablets to transfer different types of data to a repository where it can be viewed live via a mobile phone

- Allows for the capture of images outside of the classroom which can then be sent back via Multi 
Media messaging (MMS), where the message can include not just text, but also sound, images and video

- Facilitates wireless access to the Internet when used with a laptop, hence providing Internet access to students and teachers from any location in the school

One of the major project in providing research and education resources is the national research and education network (NREN). A National Research and Education Network (NREN) is a dedicated Internet infrastructure and service provider to the research and educational communities within a country [26]. NRENs provide connectivity and services to higher education establishments (typically universities) and research institutes, national and international Communities of Practice, but can also support schools, further education colleges, libraries and other public institutes. In some cases, services may be also provided to other sectors such as government and healthcare. TERNET is the NREN for Tanzania (https://www.ternet.or.tz/).

NRENs are a vital component in e-Learning, e-Science and e-Research strategies as they bring a common approach to the coordination and deployment of national and international communication networks and services. NRENs can provide a wide range of services including, for example, networks and connectivity, middleware (security, authentication and mobility, cross-institute federated support for national and international education and research), networked collaboration services for e-Learning, e-Science and e-Research, and general support services including training, dissemination and international project development. For example, Eduroam (https://www.eduroam.org/) is possibly the most well-known mobility service that is commonly provided internationally as part of NREN services.

NRENs can come together to form regional research and educational networks. Example here include West and Central African research and education network (WACREN http://wacren.net/), UbuntuNet (https://www.ubuntunet.net/), and Latin American Cooperation of advanced networks (RedCLARA - http://www.redclara.net/index.php/en/) and Arab States research and education network (ASREN http://www.asrenorg.net/). The Mtwango Coderdojo which is reported in the section three used information which is available in the NRENs.

\section{THE MTWANGO CODERDOJO PROJECT}

The work reported in this paper leverages on existing mobile, network infrastructure and does not require always on connectivity. The ability to work offline and in places where there is limited or no network connectivity is major advantage of this project compared to other similar projects. This enables students/participants to provide/share resources online and offline. It is important to realize that there are a lot of research and practical implementation to solve this problem. However, most of these implementation require either availability of reliable bandwidth or network connectivity.
The project reported in paper is a community project in Tanzania, called the Mtwango CoderDojo project. The Mtwango CoderDojo project is a community based project run by a group of volunteers to teach programming at Mtwango village - Njombe region to teen girls. Most of the girls came from the three primary schools at Mtwango village. However, we encouraged form one girls from the two secondary school located in the village to take part as well. The participation policy was first come first served. This type of the project is a small capital investment, but the quality of material and results obtained are encouraging. It is self-support, self-financing, with little or no maintenance, designed once and used everywhere. It is important to emphasize that the project uses existing infrastructure and no new resources are required. It is scalable, reliable and resilient.

We made sure that no participants had to share the computing resources i.e., mobile phones and computers. The training had two batches and each batch of participants consisted of twelve girls. During the training each participant was assigned a computer and was encouraged to bring her own mobile phone. Those who did not have mobile phones were given mobile phones for the duration of training, which was ten days. Mobile phones were used to give the participants take home assignments in the form of quizzes and puzzles.

The programming language which was taught was Scratch [27]. This visual programming language allows learners to program using building blocks. Scratch helps users learn to think creatively, reason systematically and work collaboratively which are essential skills for life in the $21^{\text {st }}$ century. We chose this language because it is an open source and there is a large community of users online for support. Reading materials including books were available in every computer and where possible shared on participants mobile phones.

The methodology of teaching was hands on and participants were encouraged to work in groups. For every new topic/concept introduced an instructor gave a talk of at most ten minutes followed by a demonstration. Then participants tried the same example with assistance from the instructor and the demonstrators. After that one or two similar tasks were given to participants.

It must be noted that while all the teaching materials were written in English, most of the communication in class were in Swahili and in some cases in the tribal language Kibena. The advantage of using other languages apart from English was that it made the girls feel comfortable, interact and ask more questions in topics/concepts they did not understand. It was possible to use the tribal language as a medium of instruction because all demonstrators came from the local secondary schools and could speak the language.

The cost of running the programme were kept at a minimum by using resources that were contributed and participants were encouraged to use their own mobile phone. Using participants own mobile phones had the advantage of them realizing that their device had more use than making calls and that it is an educational tool. It allowed the girls to continue with training longer after the formal contact period ended. Further, demonstrators were not paid, instead we organized a 
two days boot camp in secondary school Mathematics which was well received.

All teaching materials we prepared for the training were in English, this proved to be stumbling block, before we resorted to using Swahili and Kibena. Thus we are now writing some teaching materials in Swahili. Connectivity proved to be a formidable challenge, therefore, all materials were shared offline.

\section{THE PROPOSED SYSTEM}

The system proposed in this project is an integration of several existing systems put together with a view of providing high quality education content and services anywhere and anytime. Since the constituent parts of the proposed system are operating independently, in some cases the effort of getting

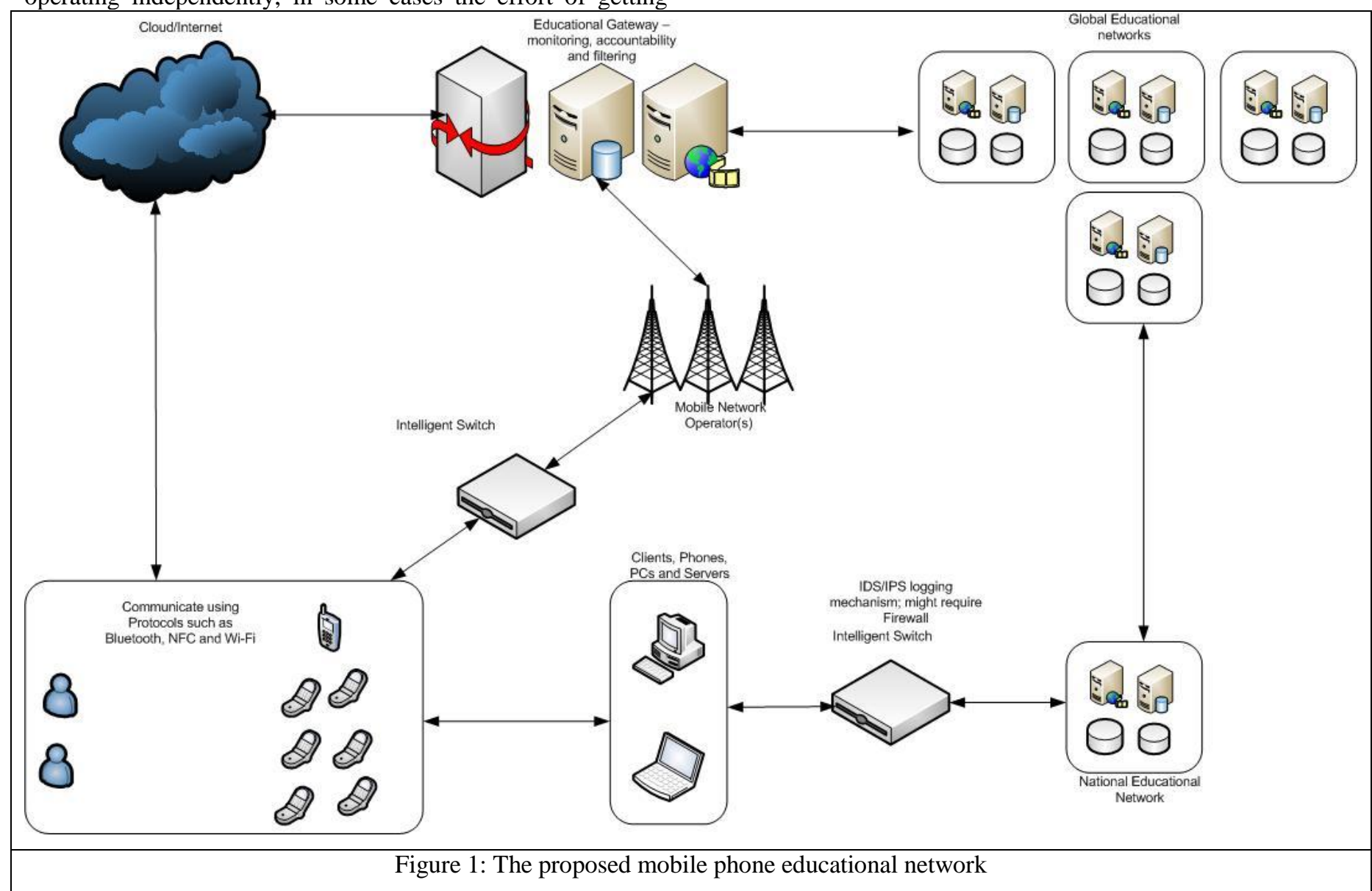

them to work together can be monumental. In those cases salient features of the system are adopted and implemented.

The central part of the system is a mobile phone which is connected to a mobile phone operator. A significant portion of the world population is either connected or can be reached by mobile phone with reasonable efforts. In places where mobile phone operators cannot reach, then the offline mode of the system is invoked. This mode is also used in places where the mobile phone connectivity is not good or disconnected due to systems not having power.

The system also can connect to the NRE in the country, regional or global. In order to reduce the cost of connecting to the mobile phone operator the system uses $\mathrm{Wi}-\mathrm{Fi}$ or other mobile phone protocols to access resources.
The proposed system covers a lot of areas of interest in using mobile phone in education. This project implemented only a small part of the proposed system. For example, the connection to NRENs was very minimum as it was considered to be a bit advanced for the target group.

It will be interesting in the future to implement middleware that will enable different similar projects to share and exchange information. This exercise may require a coordination of the Government. However, it has the potential of providing access to educational materials to Tanzanians of all walks.
In this project the issues such as ownership, controlled and monitored access, accountable access (fair use policy) and copyright of the materials used for training is not addressed. An assumption is made of implicit agreement from the author(s) of training materials. Restrictions on ownership and copyright materials can be enforced at the systems level by extending the proposed system. The same applies to payment options for access training materials.

The dependence of Tanzanians on mobile broadband for the provision of internet access is worrying and may damage the long term affordability of connectivity. This reliance is made worse by the fact that in some cases mobile broadband is 
the only alternative making using mobile phone in education costly.

\section{SUMMARY AND CONCLUSIONS}

Our research and most of the literature show that mobile phones impact educational outcomes by facilitating increased access, however, there much less evidence showing how mobiles phones impact educational outcomes by promoting new learning. The research shows that mobile phones can reduce barriers to education while attaining educational outcomes that are, at minimum, comparable to those of traditional educational methods. Our finding clearly show that learners found the interactive and the immediate feedback that mobile phone make possible appealing. The interaction is bidirectional as it enable end users collect information from their context, share it with other users and their instructors. The instructors saves the information for evaluation and further improvement of the course.

There are issues that must be addressed for mobile phones to be a central tool in increasing social inclusiveness such as most instructional materials are in English and not in native languages (Swahili or tribal languages), screen size, cost, and unfamiliarity with advanced mobile phone functions, sharing and personalization of the material. The new advances in machine learning, internet of things, data analytics, big data and artificial intelligence will make it possible to offer instructional materials based on learner capability.

Our research show the use of common, low end mobile devices for learning positively affects motivation and engagement among end users. In addition mobile phones allow a wide variety of practices: individual/group, synchronous/asynchronous, face-to-face/distance, with or without assessment. In the long run usage of mobile phones fosters the development of collaborative practices and ability to work in interdisciplinary teams.

The use of mobile phones in education leads to generation and collection of large amounts of data. This data can be analyzed using data analytics techniques to provide feedback, quality of service, uncover insights, personalized instructions and provide improvements in the applications. However, substantial challenges have to be overcomed such as data privacy concerns, security and accountability for acting upon the information gathered. Other challenges include hardware concerns related to adequate battery life and biohazard safety.

Investing in mobile phone research and development will place countries like Tanzania at the fore front of innovation. This is the case because mobile phones are disruptive technologies which have lowered the entry point, by providing a general platform for research and innovation. The number of different and fragmented projects we are witnessing now in Tanzania are a health sign of future innovation. We should expect to see innovative mobile phone applications from all walks of life. This future will be realized by smartphones.

All technology related projects and mobile phone are no exception must consider the costs and manpower necessary for upkeep, maintenance and repair and often security from outset; regardless the limitations of the budget, these expenses cannot be ignored.

Finally, it is important that the role of mobile phones in education is not over emphasized at the expense of other educational investments. Mobile phones are just one piece of the puzzle in offering relevant and appropriate education to leaners. Students and instructors need technical, logistical, and pedagogical support for incorporating and integrating mobile phones in formal and informal learning environments.

\section{REFERENCES}

[[1] J. Poushter; and R. Oates; "Cell Phones in Africa: Communication Lifeline," Pew Research15 April 20152015.

[2] J. Poushter;, J. Bell;, and R. Oates;, "Internet Seen as Positive Influence on Education but Negative on Morality in Emerging and Developing Nations " Pew Research19 March 2015.

[3] DataVision. (2016, 20 June). DataVision International Limited. Available: http://www.datavision.co.tz/index.php

[4] Selcom. (2016, 20 June). Selcom Wireless. Available: http://www.selcom.net/

[5] Maxcom. (2016, 20 June ). MaxMalipo. Available: http://www.maxcomafrica.com/

[6] B. Chipangura, "Categorizing the Provision of Mobile Centric Information Access and Interaction for Higher Educational Institutions " presented at the SAICSIT 2013, East London, South Africa, 2013.

[7] G. S. Oreku and F. J. Mtenzi, "A Review of e-Government Initiatives in Tanzania: Challenges and Opportunities," in Handbook of Research on E-Government in Emerging Economies: Adoption, E-Participation, and Legal Frameworks, K. J. Bwalya and S. F. C. Zulu, Eds., ed: IGI, 2012, p. 1020.

[8] L. Hosman; and M. Cve, "Technology, Teachers, and Training: Combining Theory with Macedonia's Experience," presented at the ICTD2010, London, UK, 2010.

[9] K. Toyama, "The Internet and Inequality," Communications of the ACM, vol. 59, pp. 28-30, 2016.

[10] J. Li. (2016, 06 September). Back to school: How young is too young for a cellphone? Available: http://www.cbc.ca/news/canada/cellphonechildren-schools-health-education-1.3745874

[11] L.-P. Beland and R. Murphy, "Communication: Technology, Distraction and Student Performance," London School of Economics CEP Discussion Paper No 1350, 2015.

[12] (2014, 06 September ). Too much screen time creates health risk for children: Health concerns include eye problems and sleep loss. Available: http://www.cbc.ca/news/canada/saskatoon/too-much-screentime-creates-health-risk-for-children-1.2753497

[13] M. Kwong. (2015, 06 September ). Smartphone, tablet overuse among toddlers may stunt development. Available: http://www.cbc.ca/news/technology/smartphone-tablet-overuse-amongtoddlers-may-stunt-development-1.2940848

[14] P. Dakin. (2014, 06 September). Social media affecting teens' concepts of friendship, intimacy. Available: http://www.cbc.ca/news/health/socialmedia-affecting-teens-concepts-of-friendship-intimacy-1.2543158

[15] W. P. Mtega;, R. Bernard;, A. C. Msungu;, and R. Sanare;, "Using Mobile Phones for Teaching and Learning Purposes in Higher Learning Institutions: the Case of Sokoine University of Agriculture in Tanzania," in The 5th UbuntuNet Alliance Annual Conference, 2012, pp. 118-129.

[16] Microsoft. (2014, 20 June ). Nokia Mobile Mathematics. Available: https://math.microsoft.com/Account/Login?ReturnUrl=\%2Ftz

[17] T. O. V. Library. (2016, 20 June). Tanzania Online Virtual Library. Available: http://www.tovl.ac.tz/

[18] NurturingMinds. (2015, 19 June 2016). Mobile Phones Mean Success for Female Students in Tanzania. Available: http://www.aidforafrica.org/uncategorized/mobile-phones-ensuresuccess-female-students-tanzania/

[19] Yoza. (2016, 20 June). The Yoza Project. Available: https://m4lit.wordpress.com/about-the-project/ 
[20] S. Srivastava. (2016, 17 June 2016). Indian Teacher Education Company EDIMPACT Launches SMS Learning for Rural Areas. Available: http://www.digitaljournal.com/pr/2975479

[21] Q. Chen; and Z. Yan;, "Does Multitasking with Mobile Phones Affect Learning? A Review," Computers in Human behavior, vol. 54, pp. 34 42, 20162016.

[22] F. Lepe-Salazar, "A Model to Analyze and Design Educational Games with Pedagogical Foundations," presented at the ACE'15, Iskandar, Malaysia, 2015.

[23] D. York; and Z. Xi; "Sharing the Knowledge," presented at the CHI 2011, Vancouver, BC, Canada, 2011.

[24] S. I. Herrera;, M. C. Fennema;, M. I. Morales;, R. A. Palavecino;, J. E Goldar;, and S. V. Zuain, "Mobile Technologies in Engineering Education," in International Connference on Interactive Collaborative Learning (ICL), Florence, Italy, 2015.

[25] N. C. f. T. i. Education, "NCTE Advice Sheet 31 - Mobile Phones," National Centre for Technology in EducationNovember 20082008.

[26] J. DYER, "The Case for National Research and Education Networks (NRENs)," TERENA2009.

[27] J. MALONEY;, M. RESNICK; N. RUSK;, B. SILVERMAN; and E. EASTMOND;, "The Scratch Programming Language and Environment," ACM Transactions on Computing Education, vol. 10, pp. 1-16, 2010. 